\title{
On output measurements via radiation pressure
}

Leeman, S.; Healey, A.J.; Forsberg, F.; Jensen, J.A.

Published in:

Proceedings of the IEEE 1990 Ultrasonics Symposium

Link to article, DOI:

10.1109/ULTSYM.1990.171594

Publication date:

1990

Document Version

Publisher's PDF, also known as Version of record

Link back to DTU Orbit

\section{Citation (APA):}

Leeman, S., Healey, A. J., Forsberg, F., \& Jensen, J. A. (1990). On output measurements via radiation pressure. In Proceedings of the IEEE 1990 Ultrasonics Symposium (pp. 1389-1392). IEEE. https://doi.org/10.1109/ULTSYM.1990.171594

\section{General rights}

Copyright and moral rights for the publications made accessible in the public portal are retained by the authors and/or other copyright owners and it is a condition of accessing publications that users recognise and abide by the legal requirements associated with these rights.

- Users may download and print one copy of any publication from the public portal for the purpose of private study or research.

- You may not further distribute the material or use it for any profit-making activity or commercial gain

- You may freely distribute the URL identifying the publication in the public portal

If you believe that this document breaches copyright please contact us providing details, and we will remove access to the work immediately and investigate your claim. 
It is shown, by simple physical argument, that measurements of Intensity with a radiation pressure balance should not agree with those based on calorimetric techniques. The conclusion is ultimately a consequence of the circumstance that radiation pressure measurements relate to wave momentum, while calorimetric methods relate to wave energy. Measurements with some typical ultrasound fields are performed with a novel type of (PVDF) hydrophone, and these allow an estimate to be made of the magnitude of the discrepancy to be expected between the two types of output measurement in a typical case.

\section{INTRODUCTION}

A travelling acoustic wave, whether continuous or pulsed, carries both energy and momentum. The former entity is gradually depleted in a lossy medium, and reveals its presence by the heating of that medium. The momentum carried by the wave is strikingly revealed by the presence of (radiative) forces exerted on an obstacle placed in its path. The energy flux of the wave is generally measured as its 'intensity', while the momentum flux is assessed via the measurement of the 'radiation pressure'. While there is no confusion about the definition of intensity, there has, in the past, been some considerable debate, and even controversy, surrounding not only the concept of radiation pressure Itself, but also the quantitation of its relationship to the intensity of the wave.

Much of the controversy was first resolved in a treatment of the problem by Livett et al. [1981], and their approach will be followed here. A number of equivalent definitions for the radiation pressure exist, but it is appropriate to adopt the relatively well-known conclusion that the radiation pressure is the time-averaged momentum flux of the wave. However, it is not entirely straightforward to relate the radiation pressure to the intensity of the wave, and additional approximations may have to be made in order to achieve a quantitative result.

It is generally assumed that the intensity of a travelling wave field can be measured either via calorimetric methods (whereby the time-averaged energy flux of the field is directly accessed), or via radiation force on an accurately specified object (from which the radiation' pressure, which is a field quantity, independent of the presence or type of object, can be assessed). If the latter pathway is chosen, then a number of additional mathematical and physical hurdles need to be surmounted in order to establish the relationships between the radiation force, radiation pressure, and Intensity. In general, such arguments tend to be limited to one-dimensional fields [Livett et al., 1981].
A (somewhat hypothetical) case of particular interest, is that of a plane travelling wave, harmonic at the origin, impinging orthogonally on a perfect (planar) absorber which intercepts the entire beam. If the absorber is sufficiently thick for the wave to be entirely absorbed within it, then the radiation pressure is equal to the radiation force per unit area of absorber, viz. $\mathcal{F}$. Moreover, it turns out that, to second order in the density perturbation, $\mathcal{F}$ is proportional to the time/space average of the wave's energy density, viz. $\mathcal{E}$ :

$\mathcal{F}=\alpha \mathcal{E}$

where $\alpha$ is a constant which depends on the equation of state of the supporting (lossless) medium [Beyer, 1978; Livett et al., 1981]. In Eq. (1), $\mathcal{F}$ represents an entity that is measured via a radiation pressure balance, while $E$, which relates to the intensity of the field, can be measured directly via calorimetric methods.

Eq. (1) provides the basis for a radiation pressure balance measurement of field intensity, and there is general agreement that the result would be identical to the more direct calorimetric approach, provided that the constraints required for the derivation of (1) were met, and provided that the propagation medium was sufficiently well characterised for a to be accurately known. It can be shown that this conclusion would not be altered in the case of a (one-dimensional) pulsed wave. However, it is the intention of this paper to point out, in a descriptive and non-mathematical way, that the essential identity of the radiometric and calorimetric assessments of intensity is bound up with the one-dimensional nature of the wave, and can not be maintained when measuring 'real' three-dimensional fields.

\section{THREE DIMENSIONAL FIELDS}

Consider a spatially bounded (pressure) pulse propagating through some uniform medium. At time $t=t_{0}$ the pulse fills a volume in space, and it is presumed that the values of the pressure throughout that volume are known, and given by the function $P(r)$, where $r$ denotes the position vector. It is immaterial whether the pulse propagates linearly or otherwise, and it is always possible to formally write the 'time-zero' pulse in the Fourier domain as:

$P(\mathbf{r})=\int d^{3} \mathbf{k} F(\mathbf{k}) \exp \{-i \mathbf{k} \cdot \mathbf{r}\}$ 
For linear wave propagation in a lossless, dispersionless medium with wave velocity $c$ it is easy to establish that the pulse at any subsequent time may be formed from $F(\mathbf{k})$ via:

$P(\mathbf{r}, t)=\int d^{3} \mathbf{k} F(\mathbf{k}) \exp \{i \omega(\mathbf{k}) \tau-i \mathbf{k} \cdot \mathbf{r}\}$

with $\omega(\mathbf{k})=c|\mathbf{k}|$, and $\tau=\left(t-t_{0}\right)$. This is nothing more than the well-known decomposition of a travelling pulse into a superposition of travelling plane waves, of different frequencies and propagation directions [Leeman et al., 1985].

The extension of Eq. (3) to the case of a linear, lossy medium is also uncomplicated [Leeman et al., 1985]. It is merely necessary to replace the set of travelling harmonic plane waves by a set of appropriately attenuated plane waves. Including velocity and attenuation dispersion is achieved in a similar fashion, and is thus relatively straightforward. Thus, once the 'time-zero' Fourier decomposition of the field is known, the field can be evaluated at other times and locations by the simple expedient of allowing each plane wave component of the initial decomposition to propagate according to the law dictated by the characteristics of the ambient medium (as encoded in a wave equation, for example).

It is perhaps not so readily appreciated that the same artifice outlined above may also be applied to the description of nonlinearly propagating bounded fields. In this case, each plane wave component propagates according to the nonlinear law dictated by the supporting medium. The 'time zero' wave components which point in any one particular direction unfold in space and time exactly as would a (hypothetical) true one-dimensional pulse, with the same initial one-dimensional spectrum, which propagates in the same (nonlinear) medium. All the familiar (one dimensional) nonlinear propagation features [Pierce, 1981] wilt be exhibited by the subset of wave components pointing in any one particular direction. However, it is important to appreciate that the decomposition in Eq. (3), when applied to nonlinear fields, implies that no interaction (even nonlinear) occurs between subsets of wave components which point in different directions. This contention is supported by the experimental results reported in an accompanying paper [Forsberg et al., 1990], and no attempt is made here to provide a more formal proof.

\section{INFLUENCE ON MEASUREMENT}

The relevance of the above considerations for the measurement of field intensity may be easily demonstrated. For simplicity, an axially symmetric field is assumed, orthogonally incident on an ideally absorbing planar target of a radiation pressure balance. The radiation force contributed by the wave component that is perpendicular to the plane of the target is related to the mean energy density of that component by an expression such as Eq. (1). However, a modified relationship holds for the components which impinge on the target at an angle $\theta$, as schematically illustrated in Fig. 1. In this case, the radiation pressure carried by each component is at an angle $\theta$ to that carried by the perpendicular component. However, because of the angular symmetry of the beam, only the component of the radiation pressure that is perpendicular to the target actually contributes to the radiation force, since the tangential components cancel out, as shown in Fig. 1. Thus, for these components, the radiation force and mean energy density are related via
$\mathcal{F}_{\theta}=\alpha \mathcal{E}_{\theta} \cos \theta$

where the subscript, $\theta$, denotes the wave component that enters the target at the indicated angle.

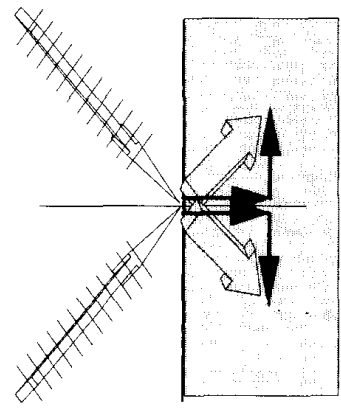

Figure 1. Schematic representation of the cancellation of the tangential components of the radiation force of the angular wave components of an axially symmetric field.

While the contribution to the total radiation force by the angular wave components is reduced, their mean energy density would contribute fully to a measurement made by a calorimeter (which measures the energy contributed by each component that enters the device, irrespective of the angle of incidence). Clearly, the field intensities as measured by the two techniques can not be expected to be identical, unless the radiation force measurement can be corrected for the reduced contribution of the angular components, as expressed in Eq. (4). Note that this observation holds irrespective of the axial symmetry of the beam, as assumed in the above discussion.

\section{MAGNITUDE OF THE PROBLEM}

The magnitude of the discrepancy between radiometric and calorimetric assessments of field intensity can be established if the angular decomposition of the measured field can be ascertained. This is a rather more subtle problem than appears at first glance, but it can be achieved by utilising an effectively 'infinite', planar, uniformly sensitive hydrophone for field measurement. In this context, 'infinite' means that the hydrophone aperture is larger than the effective diameter of the beam. The underlying theory of such a measurement has already been presented [Leeman et al., 1985], and a practical device, based on a thin (approx. 25u) uniformly poled and electroded PVDF membrane, has been constructed and utilised for a number of measurements [Costa et al., 1988]. The hydrophone essentially acts as a filter which responds only to those wave components that are orthogonal to the plane of the PVDF membrane. Thus, by measuring the field with the plane of the hydrophone oriented at various angles to the beam direction, the full three-dimensional 'zero-time' spectrum of the pulse can be mapped.

One such set of measurements is shown in Figs. $2 \& 3$, which show different representations of the power spectra of the wave components lying in a plane through the central axis of the beam (denoted by $\theta=0$ ). In this case, the (pulsed) field from the transducer (Philips $2.25 \mathrm{MHz}, 19 \mathrm{~mm}$ diameter, $9 \mathrm{~cm}$ focus) exhibits a 
good degree of axial symmetry. Note also that this transducer is very directive, with the angular spread of the wave components somewhat less than $\pm 15^{\circ}$. The excitation voltage was maintained at a level that ensured linear field transmission: under these circumstances the output of the hydrophone is invariant with distance from the transducer [Costa et al., 1988]

Given the angular dependence of the power spectra of the wave components, it is also possible to estimate the error in the radiometric intensity assessment. For the tield depicted in Fig. 2, a radiation pressure balance with an ideally absorbing target (which, in turn, cannot be realised without generating unwanted reflections [Livett and Leeman, 1983]) would under-estimate the intensity by less than $1 \%$. The effect is indeed rather small for a transducer as directive as the one shown here, but will be greater for smaller diameter or more heavily focused transmitters, or with different types of radiation pressure balance targets. But it is worth pointing out that, in principle, such a discrepancy exists.

\section{IMPLICATIONS AND CONCLUSIONS}

The results reported here have obvious implications for the measurement of transducer output by radiometric methods. However, there are also somewhat more general conclusions to be drawn. The 'zero-time' spectrum can be measured at any convenient location in the field, provided that a sufficiently large aperture hydrophone, as described above, is available. Provided that the propagation properties of the supporting medium are known, the time variation of the field at any other location can then be predicted, and computed, via integral expressions of the type indicated in Eq. (3). As the subsequent discussion showed, such predictions can be made even for nonlinear fields. Such an approach is considered to be particularly useful when measuring or monitoring, output from very highly focused transmitting systems, such as lithotripters. Where the focal point intensities are sufficiently high to possibly damage any physical device placed there, large aperture measurements may be safely made at a relatively low intensity location, and focal point values obtained by computation. While great experimental accuracy will be called for, particularly in measuring the phase of the 'zero-time' spectrum, the advantage of using (large aperture) thin PVDF membrane hydrophones is that they may be used also to measure, in a relatively artefact-free manner, the propagation properties of the (liquid) supporting medium [Costa and Leeman, 1989]. The results presented above aiso indicate that, for relatively directive fields, a large aperture (thin film) hydrophone can be oriented to monitor a substantial fraction of the total output of a transmitter, and can be permanently placed in the path of the beam with little distortion of the field beyond: the relative 'transparency' of such hydrophones has already been demonstrated and exploited for the measurement of ultrasound reflectivity [Costa and Leeman, 1989]. It should also be apparent that large aperture hydrophones have a role to play in the calibration of pulsed fields and the assessment of transducer quality.

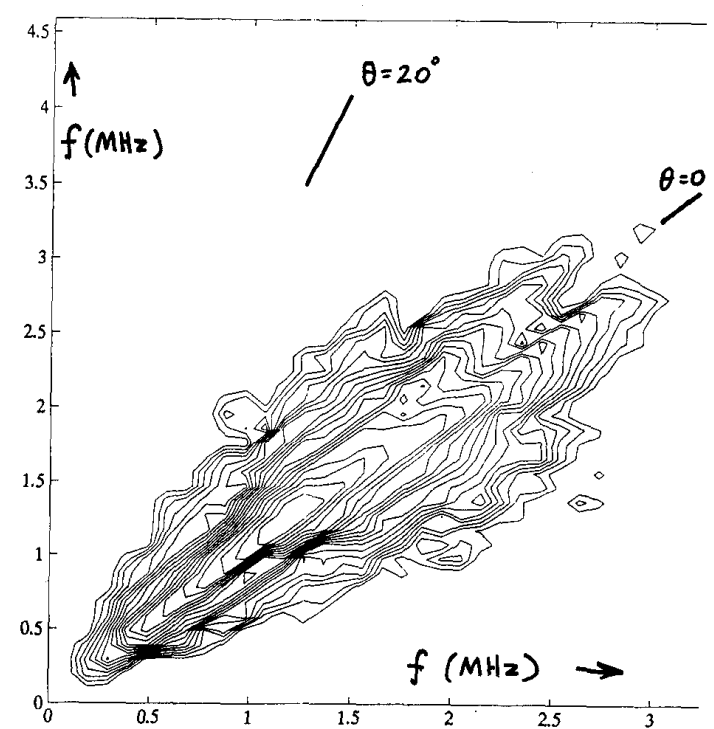

Figure 2. Contour plot of a two dimensional slice through the three-dimensional log power spectrum of a typical medical ultrasound pulse.

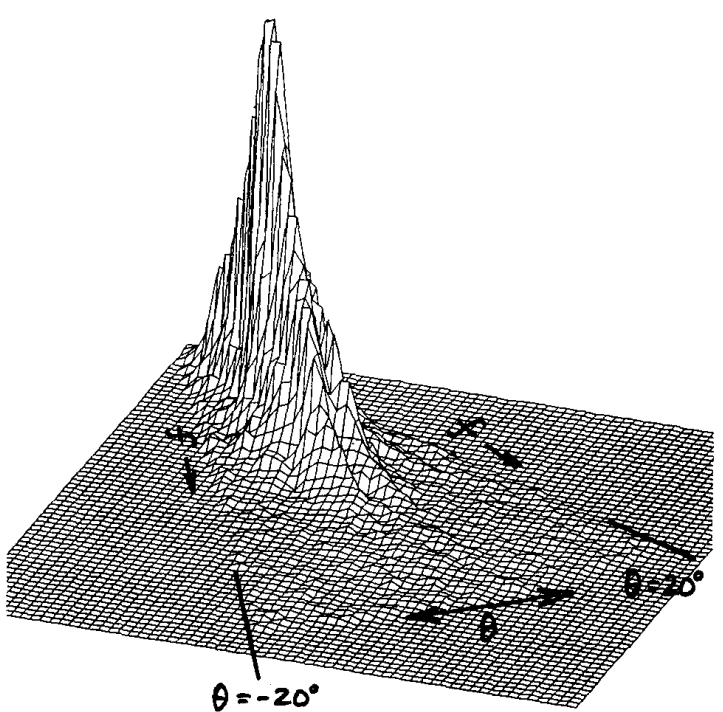

Figure 3. Isometric view of a two dimensional slice through the three-dimensional amplitude spectrum of a typical medical ultrasound pulse.

1990 ULTRASONICS SYMPOSIUM - 1391 


\section{ACKNOWLEDGEMENTS}

The work reported here would not have been possible without the generous and appreciated support of the Wellcome Trust, who are thanked also for providing a travel grant to enable presentation of this communication.

\section{REFERENCES}

Beyer, R.T., 1978, "Radiation pressure - history of a mislabeled tensor", J. Acoust. Soc. Am., 63, pp. 1025-1030.

Costa, E.T., Leeman, S., and Hoddinott, J.C., 1988, "A new approach towards measurement of three-dimensional puises: linear and nonlinear fields", In: Physics in Medical U/trasound II. Eds: Evans, D.H.and Martin, K. [IPSM, York (UK)], pp. 87-93.

Costa, E.T. and Leeman, S., 1989. "Diffraction artefacts and their removal". In: Acoustical Imaging, 17, Eds.: Shimizu, $H_{\text {., Chuba- }}$ chi, N., and Kushibiki, J. [Plenum Press, New York], pp 403-411. Forsberg, F., Healey, A.J., Leeman, S., and Costa, E.T., 1990. "Measuring nonlinear fields without diffraction correction". In: Proc. 1990 IEEE Ultrasonics Symposium, Ed.: McAvoy, B.R. [IEEE, New York].

Leeman, S., Seggie, D.A., Ferrari, L.A., Sankar, P.V., and Doherty, M., 1985. "Diffraction-free attenuation estimation". In: Uitrasonics International 85, Ed.: Novak, Z. [Butterworth, Guildford], pp. 128-132.

Livett, A.J., Emery, E.W., and Leeman, S., 1981. "Acoustic radiation pressure", J. Sound and Vibration, 76(1), pp. 1-11.

Livett, A.J. and Leeman, S., 1983. "Radiation pressure and its measurement". In: Proc. 1983 IEEE UItrasonics Symposium, Ed.: McAvoy, B.R. [IEEE, New York] pp. 749-752.

Pierce, A.D., 1981. Acoustics: an introduction to its physical principles and applications [McGraw-Hill, New York]. 